\title{
Prof. Abderhalden über Soziale Hygiene, allgemeine Therapie, Naturheilkunde und Homöopathie.
}

\author{
Von Prof. Friedrich Schultze in Bonn.
}

Die Ausführungen, die der bekannte Hallenser Physiologe Herr Prof. Abderhalden in der Sitzung der preußischen Landesversammlung vom 23. Mai 1919 gemacht hat, können leider nicht in allen Teilen unwidersprochen bleiben. Sie liegen jetzt in stenographischer Wiedergabe vor und dienten der Begründung mehrerer Anträge des Ausschusses für Bevölkerungspolitik, dessen Wortführer Herr Prof. Abderhalden war.

Der erste Antrag betrifft den Universitätsunterricht in ,So. zialer Hygiene“. Er ersucht die Staatsregierung, sowohl Lehrstellen, als auch Forschungsstätten für ,,Soziale Hygiene" zu gründen.

Herr Prof. Abderhalden behauptet gleich im Anfange seiner Rede, daß von dem großen Gebiete der gesamten Hygiene ,bis zur Zeit" ein sehr wichtiger und großer Teil, nämlich die sogenannte Soziale Hygiene, an den Universitäten nicht berücksichtigt werde. Nur einzelne Professoren und namentlich Privatgelehrte und Gesellschaften hätten diesen Zweig der Hygiene gepflegt. Die Studierenden der Medizin hätten aber gar keine Gelegenheit, dieses außerordentlich wichtige Gebiet „, im Zus ammenhange" kennen zu lernen.

Die im ersten Satze ausgesprochene Behauptung, daß die Soziale Hygiene überhaupt nicht berücksichtigt würde, wird also in diesem Nachsatze bedeutend eingeschränkt. Denn nach ihm ist doch nur die Art des bisherigen Unterrichtes nicht zweckmäßig.

Im weiteren Verfolg seiner Auseinandersetzungen erklärt Herr Prof. Abderhalden, was er unter ,Sozialer Hygiene" versteht. Sie beschäftigt sich mit dem Einflusse der ,,sozialen" Umwelt auf die Gesundheit des Menschen. Es werden in ihr die $\mathrm{Be}$ rufskrankheiten behandelt, die Gewerbeh'siene, der Einfluß der Wohnstätten auf den Menschen, der Schule auf das Kind, ferner das Fürsorgewesen, das gesamte Kranken. kassenwesen, die ganze Krankenversicherung, die Altersversicherung und alle Ernährungsfragen.

Von diesen Gegenständen wird nun unzwelfelhaft ein großer Teil seit Pettenkofer von den Ordinarien der Hygiene überall gelesen und dabei selbstverständlich der Einfluß der "sozialen" Umwelt auf den Menschen in bezug auf Beruf, Wohnung, Wasser, Luft usw. sowohl auf den Einzelnen, als auch auf die Gesamtheit in Betracht gezogen. Hier in Bonn wurde wenigstens stets und wird auch jetzt die Gewerbe-, Berufs-, Schul-, Wohnungs-, Wasserhygiene ausgiebig berücksichtigt. Herr Prof. Ne umann hat sogar für dieses Semester hygienische Besichtigungen und für das nächste Semester, entsprechend der neueren Bezeichnung, Hygiene und Soziale Hygiene angekündigt. Ebenso wird von ihm eingehend über Ernährungsfragen vorgetragen.

Ueber das gesamte Versicherungswesen und die Begutachtung von bezüglichen Krankheitsfällen trägt schon seit vielen Jahren bei uns in Bonn Herr Prof. Ru mpf theoretisch und praktisch vor, neben ihm in letzter Zeit noch Herr Dr. Horn, und auch in der Medizinischen Klinik haben schon seit mehr als zwei Jahrzehnten praktische Kurse über Unfallbegutachtung stattgefunden.

Die Behauptung Abderhaldens, daß ,an den Universitäten die sogenannte Soziale Hygiene nicht berücksichtigt wird" ist also jedenfalls für Bonn völlig unrichtig.

Den Ausführungen des Herrn Prof. Abderhalden stimmte die Landesversammlung, wie schon vorher der Ausschuß, einstimmig zu, und auch der Herr Minister äußerte sich mit schmerzlichem Blicke auf ,,die außerordentlich traurige" Lage der Finanzen sehr ,sympathisch".

Der zweite Antrag des Herrn Prof. Abderhalden und des Ausschusses für Bevölkerungspolitik betraf ,,die Erweiterung des Unterrichts in der allgemeinen "Therapie“ 
Mit Recht hält der Antragsteller selbst diesen Antrag, ,dell theoretischen und praktischen Unterricht in der allgemeinen Therapie $\mathrm{zu}$ erweitern und die Erforschung ihrer Wirkung $\mathrm{zu}$ vertiefen", für nicht unbedingt klar.

Er erläutert ihn zunächst durch die Forderung, daß ,die Ausbildung der Aerzte ganz bedeutend verbessert werden muß, und zwar vor allem nach der Richtung hin, daß der Arzt so ausgebildet wird, daß er nicht nur Krankheiten, sondern vor allen Dingen den Kranken zu behandeln erlernt". Das hat bekanntlich friher besonders Leyden oft genug ausgesprochen, und sicher hat wohl jeder Kliniker, wie ich selbst, in seinen Vorlesungen darauf hingewiesen, daß um jedes einzelne Organ, den Magen oder den Uterus herum $\mathrm{dc} h$ noch so vieles andere liege, was auch berücksichtigt werden müsse. Daß vor allem auch die Seele des Menschen berücksichtigt werden müsse. Stets haben vor allem die Psychiater das Seelische betont, und in seinen ,Bemerkungen über Mängel in der ärztlichen Vorbildung" hat der Innere Kliniker Stursbergin ${ }^{1}$ vor kurzem eindringlich darauf hingewiesen, daß schon viel früher als bisher der Medizinstudierende an den kranken Menschen herangeführt werden müsse durch eine Pflichtvorlesung in den ersten Semestern über Krankenpflege mit möglichst ausgiebigen praktischen Uebungen.

Geschehen ist also seitens der Kliniker nach der von Abderhalden gewünschten Richtung schon Vieles, was wohl eine Erwähnung verdient hätte. Daß gerade die bedeutende Verbesserung des ,biologischen Denkens, d. h. der Ausbildung in der gesamten Physiologie“" zu dem erwähnten Ziele führt, wie Abderhalden meint, bleibt fraglicher, man müßte denn die Ausbildung in der Psychologie, und zwar besonders in der praktischen Seelenkunde, unter den Begriff der Physiologie unterbringen.

Weiterhin behauptet dann Abderhalden zur Begründung seines Antrages, daß die sogenannte Schulmedizin sich gegen die Naturheilmethoden von vornherein ablehnend verhalten habe. Sie habe das Gute zu nehmen, wo es zu finden ist; auch ein Laie könne ohne Fachaubbildung etwas Hervorragendes entdecken. Diese Behauptung Abderhaldens wird Wasser auf die Mühlen der Naturheilkünstler und vieler Pfuscher sein, von deren Gehässigkeit im Kampfe gegen uns Aerzte wohl der Hallenser Physiologe nicht hinreichend Kenntnis hat. - Schon der Name "Naturheilkunde“, der gegen die Aerzte zu hetzerischen Zwecken als Angriffsmittel erfunden wurde, muß beanstandet werden. Sind denn etwa die Digitalisblätter, die die Aerzte verwenden, unnatürliche oder übernatürliche Mittel, oder kommt Arsenik nicht in der Natur vor, sondern außerhalb derselben? Und wirkt nicht umgekehrt ein physikalisches Mittel, wie kaltes oder heißes Wasser, oft genug bei Kranken recht schädlich, ebenso wie unzweckmäBig angewandte Luft- und Sonnenbäder? Ich meine, Herr Prof. Abderhalden hätte das Wort ,Naturheilkunde" um so weniger brauchen sollen, als er durch Umfragen selber festgestellt hat, was jeder Kliniker längst wei $\beta$, daß das Interesse für die sogenannten physikalischen Methoden sehr groß ist. Die Anwendung der Elektrizität ist $\mathrm{z}$, B. so alt wie der Galvanismus und die Faradisation selbst, sogar wie die Kenntnis der Reibungselektrizität. Die von Leyden und Goldscheider herausgegebene Zeitschrift für diätetische und physikalische Therapie " datiert bereits vom Jahre 1898, und das "Handbuch der Ernährungs therapie und Diätetik" von Leyden ist in Verbindung mit einer großen Anzahl von angesehenen Klinikern, als richtigen Naturheilkundigen, aber auch als Heilkundigen im allgemeinen, schon 1897 herausgegeben worden. $\mathrm{Zu}$ ihnen hatten sich von Nichtklinikern Liebreich und Rubner gesellt.

Anstatt die Verdienste dieser Männer auch nur zu streifen, wird den Universitätslehrern und Aerzten zum Vorwurfe gemacht, daß sie sich gegenüber den „Naturheilmethoden“ ablehnend verhalten hätten, allerdings zum großen Teile nicht wegen der Art der Methoden, sondern wegen der Persönlichkeiten, die sie ausübten, und vor allem wegen der einseitigen, unkritischen Anwendung dieser Methoden Die Schulmedizin ,darf aber vorgeschlagene Methoden nịcht ablehnen, sondern sie muß sie durcharbeiten, um den guten Kern der ganzen Vorschläge herauszuarbeiten". So werden wir von Abderhalden belehrt. Auch so törichte, wie die des Schäfers Ast oder die Augendiagnose Felkes? Und hat denn die ,Schulmedizin“" sich gegenuber den Verfahren, z. B. von Priessnitz oder von Schroth, anders verhalten, wie es nach Abderhalden richtig ist? Und wenn sie dabei vorsichtig tastend und kritisch vorgegangen ist, hat sie denn darin nicht recht? Handelt sie anders gegenüber neuen Heilverfahren, die aus ihrem eigenen Kreise stammen?

Als der Magnetiseur und Nichtmediziner Hansen einst Deutschland durchzog und"seine hypnotischen Vorstellungen gab, da haben weder der Physiologe Heidenhain in Breslau, noch der Physiologe $\mathrm{Kühne} \mathrm{noch} \mathrm{wir} \mathrm{Kliniker} \mathrm{in} \mathrm{Heidelberg} \mathrm{ver-}$ schmäht, die Sache zu prüfen und den ,Kern“ herauszuschälen. Wir sind dabei sogar auf die Bretter des Theaters gegangen. Und auch dieser Kern der Hypnose, die seelische Suggestion, wurde dann weiter von Klinikern ausgebaut, allerdings auch von Kurpfuschern ausgenutzt.

Die Anklage Abderhaldens vor der Landesversammlung besteht also nicht zu Recht. Auch ist es nicht richtig, daß es mit der Anwendung der physikalischen Therapie bei den Aerzten so schlecht steht: Wenigstens sagt Bier in seinem Buche über
Hyperämie als Heilmittel $^{1}$ ): „Es gibt wohl jetzt keinen Arzt, der nicht die allgemeinen hygienischen Verhältnisse, Licht, gute Luft, Ernährung für die Erhaltung und Wiedergewinnung der Gesundheit mit in die erste Linie stellte."

Wenn nun trotz dieser seit lange bestehenden Vorliebe der Universitätslehrer und der Aerzte für die physikalische und allemeine Therapie (in letzter Zeit auch besonders für die Strahlenbehandlung) Abderhalden darüber klagen muß, daß nach seinen Erkundigungen an manchen Universitäten die notwendigsten Einrichtungen für den Unterricht in der physikalischen Therapie ehlen, so ist selbstverständlich, ihm darin beizustimmen, daß baldmöglichst in dieser Beziehung Abhilfe geschaffen werden muß. Auch mir wurde seinerzeit, als ich Liegehallen für die Tuberkulösen meiner Klinik haben wollte, von einem Vertreter des preußischen Finanzministeriums gesagt: „Wozu brauchen Sie denn Tuberkulöse? Die können Sie ja doch nicht heilen." Wir haben aber n Bonn ein gut eingerichtetes Institut für physikalische Therapie

Sehr einverstanden kann man sich auch ferner damit erklären, daß der Ausschuß für Bevölkerungspolitik ,beschlossen“ hat, den Unterricht in der Allgemeinen Therapie nicht von selbstandigen Stellen außerhalb der sonstigen Kliniken erteilen $\mathrm{zu}$ lassen, sondern , innerhalb der bestehenden medizinischen Kliniken und Polikliniken" Dieser Beschluß steht allerdings im Gegensatz zu demjenigen in bezıg auf die ,Soziale" Hygiene, nämlich bei dieser den Unterricht über die verschiedenen Fürsorgestellen für Tuberkulöse, Säuglinge usw., von den einzelnen zugehörigen Instituten loszulösen, ebenso wie denjenigen des größten Teiles der Hygiene von den bestehenden Hygienisch-bakteriologischen Anstalten und Laboratorien

Zuletzt wünscht Herr Prof. Abderhalden noch auf besonderen Wunsch (wessen?), daß die Homöopathie im Universitätsunterricht ebenfalls berücksichtigt werde und „,die* Arzneimittelwirkung nach dieser Seite hin noch ausgebaut werden soll". Die Forschung habe auf dies e m Gebiete außerordentlich viel geleistet (auf dem der Homöopathie selbst? und was?)! ,Es ist uns wohl bekannt, daß bestimmte Mittel in Spuren eine vollständig andere Wirkung haben als in größeren Mengen." Vielleicht spielt Abderhald en damit auf neue. Untersuchungen über die Wirkung der Hypophysis und Aehnliches an. Was das aber mit den Behauptungen der Homöopathen zu tun hat, daß ungeheure Verdünnungen einer Unzahl von Pflanzen und Mineralien Krankheiten heilen oder bessern, ist nicht ersichtlich, - wenn wir auch wissen, daß z. B. schon sehr kleine Dosen von Tuberkulin oder von Radium gewisse Wirkungen haben können, und wenn es auch bekannt ist, daß kleinere. Dosen von gewissen Substanzen andere Wirkungen haben als größere.

Sollen wir aber deswegen gewisse Grundlagen der „homöopathischen Lehren" anerkennen, z. B. daß das Einnehmen von Chinarinde Wechselfiebersymptome macht, wie Hahnemann behauptete, oder daß die Muskelkraft, die beim Verreiben de Heilsubstanzen gebraucht wird, in die Arznei übergehe und ihr eine neue, vorher nicht dagewesene, Kraft verleihe?

Und sollte wirklich so vielen klugen Klinikern von früher, Schönlein, Skoda, Bamberger, oder später z. B. Kuss maul, die spezifische Heilkraft der ",homöopathischen Mittel" entgangen sein? Der wahre Kern der Homöopathie; nach dem wir auf den Wunsch Abderhaldens fahnden sollen und nach dem auch gefahndet wurde, ist doch wohl der, daß sie ein viel größeres $\mathrm{Ge}$ wicht auf die Allgemeinbehandlung der Kranken legte, als das zur Zeit Hahnemanns bei den damaligen Aerzten der Fall war, z. B. auch auf die Regulierung der Diät, und daß sie den übermäßigen Gebrauch von stark wirkenden Chemikalien einschränkte.

Daß somit die Homöopathie ebenso wie andere Heilmethoden, z. B. die von Rademacher, in den Vorlesungen über die Geschichte der Medizin besprochen werden, ist durchaus richtig, ebenso wie ein Hinweis auf sie besonders in der Pharmakologie oder in der Klinik zweckmäßig ist, ebenso wie auch etwa die Erwähnung des Besprechens und der Volksmedizin überhaupt. Vor allem ist es aber durchaus notwendig auf die Rolle der Suggestion und Autosuggestion bei allen diesen Dingen hinzuweisen, wie denn uberhaupt der angehende Mediziner nicht eindringlich und oft genug auf den überaus großen Einfluß dieser Potenzen für die Beurteilung der Wirkung jeder Therapie hingewiesen werden kann. Diese seelischen "Potenzen" sind wiehtiger als die Hahnemannschen. Auf diesen großen Einfluß des Seelischen bei jeder Beurteilung der Wirkung von Arzneimitteln jeder Art, seien sie physikalischer oder chemischer oder ,psychotherapeutischer" Natur, geht aber Ab derhald en leider mit keinem Worte ein. Ein Kurs besonders auch über hysterische Seelenzustände wäre für jeden angehenden Arzt und auch in Volkshochschulkursen von großem Werte. 